Experiencias en Red. Enseñanzas de los procesos de organización comunitaria durante la pandemia en barrios de Tolosa y Ringuelet para fortalecer el sistema de salud Emiliano Rimoldi, María Sofía Bernat, Pablo Barrenengoa, Sofía Malleville, Manuel Fonseca, José Eduardo Moreno, Joaquín Lazarte, Ayelen Gorza

Question/Cuestión, Nro.70, Vol.3, diciembre 2021

ISSN: 1669-6581

URL de la Revista: https://perio.unlp.edu.ar/ojs/index.php/question/

IICom -FPyCS -UNLP

DOI: https//doi.org/10.24215/16696581e598

\title{
Experiencias en Red
}

Enseñanzas de los procesos de organización comunitaria durante la pandemia en barrios de Tolosa y Ringuelet para fortalecer el sistema de salud

\section{Network experiences}

Lessons from community organization processes during the pandemic in neighborhoods of Tolosa and Ringuelet to strengthen the health system

\section{Emiliano Rimoldi}

Instituto de Investigaciones en Educación Superior; Universidad Nacional de La Plata/ Comisión de Investigaciones Científicas de la provincia de Buenos Aires/ Dirección de Redes Intersectoriales en Salud - Dirección Provincial de Hospitales.

emirimoldi@gmail.com

https://orcid.org/0000-0003-0858-4509 


\section{María Sofía Bernat}

Dirección de Redes Intersectoriales en Salud; Universidad Nacional de La Plata. sofiabernat@gmail.com

https://orcid.org/0000-0002-7178-3829

\section{Pablo Barrenengoa}

Dirección de Políticas de Atención de la Salud - Dirección Provincial de Hospitales; Ministerio de Salud de la Provincia de Buenos Aires/ Laboratorio de Psicología Comunitaria y Políticas Públicas; Facultad de Psicología UNLP. pbarrenengoa@gmail.com https://orcid.org/0000-0001-8375-1317

\section{Sofía Malleville}

Laboratorio de Estudios en Sociología y Economía del Trabajo - Instituto de Investigaciones en Humanidades y Ciencias Sociales; Facultad de Humanidades y Ciencias de la Educación; Universidad Nacional de La Plata. mallevillesofia@gmail.com http://orcid.org/0000-0003-3094-5796

\section{Manuel Fonseca}

Dirección de Redes Intersectoriales en Salud; Universidad Nacional de La Plata. 
Dirección de Políticas de Atención de la Salud - Dirección Provincial de Hospitales; Ministerio de Salud de la Provincia de Buenos Aires.

chi2.consultora@gmail.com http://orcid.org/0000-0002-7952-1196

\section{Joaquín Lazarte}

Dirección de Políticas de Atención de la Salud - Dirección Provincial de Hospitales; Ministerio de Salud de la Provincia de Buenos Aires.

jo.lazarte@gmail.com

https://orcid.org/0000-0002-3647-6196

Ayelen Gorza

Dirección de Políticas de Atención de la Salud - Dirección Provincial de Hospitales; Ministerio de Salud de la Provincia de Buenos Aires.

gorzaayelen@gmail.com

https://orcid.org/0000-0003-2888-4445 


\section{Resumen}

Este artículo presenta una sistematización de los primeros resultados de un proyecto de investigación que busca analizar las estrategias de promoción, prevención y asistencia en los barrios de Tolosa y Ringuelet (La Plata) en el marco de la pandemia de COVID-19. Dichas estrategias fueron desarrolladas a partir de la construcción de redes intersectoriales en salud y diversos dispositivos de organización comunitaria impulsados por los hospitales San Roque y Gutiérrez, la Universidad Nacional de La Plata (UNLP) y las organizaciones comunitarias.

\section{Palabras clave}

Redes intersectoriales en salud; organización comunitaria; salud colectiva; COVID-19.

\section{Abstract}

This article is based on a systematization of the first results of a research project that aims to analyze the promotion, prevention and assistance strategies in the neighborhoods of Tolosa and Ringuelet (La Plata) during the COVID-19 pandemic. These strategies were developed from the construction of intersectoral health networks and community organization devices promoted by the San Roque and Gutiérrez hospitals, the National University of La Plata (UNLP) and community organizations.

\section{Keywords}

Intersectoral health networks; community organizations; collective health; COVID-19.

Los efectos de la pandemia de COVID-19 se disiparon radicularmente, operando tanto en niveles macro como micro sociales. Se vieron alteradas las estructuras sociopolíticas y

\footnotetext{
IICom (Instituto de Investigaciones en Comunicación)

Facultad de Periodismo y Comunicación Social

Universidad Nacional de La Plata
} 
culturales y las consecuencias de dichos efectos comienzan a evidenciarse a medida que superamos los estallidos epidemiológicos y producimos mayores y mejores conocimientos colectivos sobre el comportamiento del virus. Estamos transitando una etapa de decantación de las experiencias vividas que dan lugar a sistematizaciones y reflexiones acerca de las transformaciones requeridas para avanzar hacia un nuevo modelo de ciudadanía que, sin ser uniforme, muestre un profundo compromiso con las necesidades y proyectos colectivos.

Con la pandemia se hicieron visibles las desigualdades estructurales de nuestro país y cómo éstas impactan crudamente en los sectores populares. Al mismo tiempo, emergieron esfuerzos comunitarios extraordinarios con la consciencia de que las desigualdades del hábitat ubican a ciertas poblaciones en situaciones de mayor vulnerabilidad ante el virus (Sanchís, 2020; Vommaro, 2020). Se fortalecieron procesos de organización popular, que funcionaron como "infraestructuras" (CLACSO, 2020) de las estructuras de los sistemas de salud; complementando o suplementando las iniciativas gubernamentales frente a la pandemia. Esto interpeló al campo de la salud colectiva en su conjunto. Así, quedó evidenciado una vez más que los procesos de salud-enfermedad-atención-cuidado exceden la mirada biomédica y requieren el abordaje de las problemáticas sanitarias desde una perspectiva más amplia, que incluya el enfoque de la epidemiología crítica (Breilh, 2013).

Por lo anterior, en este trabajo nos proponemos describir y sistematizar las prácticas de abordaje comunitario de la pandemia desarrolladas por hospitales, organizaciones sociales y la Universidad Nacional de La Plata en las localidades de Tolosa y Ringuelet durante la emergencia sanitaria. (1)

El territorio contempla un área que involucra a las delegaciones mencionadas $\mathrm{y}$, en particular, a los siguientes barrios populares: El Mercadito, La Unión, El Churrasco, Barrio Nuevo, La Isla y La Bajada. A pesar de sus diferencias y distintos modos de conformación, estos barrios comparten características sociodemográficas y problemáticas similares con respecto al acceso al sistema de salud, a la educación, a las modalidades de empleo y a las condiciones de hábitat (por ejemplo, en lo que respecta a servicios y a espacios públicos). De todas maneras, conviven en la zona casas enmarcadas en planes de vivienda y otras en condiciones de extrema vulnerabilidad. 
Estos barrios forman parte de las áreas programáticas (2) de los Hospitales Gutiérrez de La Plata y San Roque de Gonnet. Durante la pandemia, allí se desarrollaron diferentes prácticas y articulaciones intersectoriales con el propósito de ampliar los horizontes de complejidad e integralidad en el abordaje de la emergencia sanitaria. En particular, estas acciones se llevaron a cabo a partir de la articulación de tres grupos de actores: los Servicios de Áreas Programáticas y Redes en Salud (SAPS) de los efectores mencionados, el voluntariado universitario y la mesa barrial Tolosa-Ringuelet, un espacio conformado por instituciones y organizaciones que intervienen en este territorio. Desde una perspectiva de abordaje interdisciplinaria y un enfoque amplio de las problemáticas de salud, se realizaron aproximadamente 50 operativos sanitarios en la zona entre 2020 y 2021 con la participación de los actores mencionados. Algunos de estos operativos estuvieron estrechamente vinculados a identificar a personas que podrían tener COVID-19 y garantizar el aislamiento, como los operativos "Detectar" (3), pero en otras oportunidades se abordaron problemáticas de salud diversas. Tal es el caso de los relevamientos "casa por casa" que registraban a personas con enfermedades no transmisibles, embarazos sin controles de salud, accesibilidad a métodos anticonceptivos, situaciones de violencias de género, entre otras problemáticas. Al mismo tiempo, se realizaron postas de salud integral, donde se garantizaba el acceso a la salud a sectores de la comunidad que presentaban dificultades para hacerlo.

La articulación de estos dispositivos se vio fortalecida por la realización de 21 Núcleos Operativos de Emergencia (NOE) en la zona de Tolosa y Ringuelet. Se trató de talleres en los que se compartía información confiable y medidas de cuidado en torno al COVID-19 y a otras problemáticas como dificultades para el acceso a una alimentación saludable y violencias de género. Estos NOE implicaron la puesta en marcha de un dispositivo en tiempo real para el abordaje territorial de la pandemia junto a comités de crisis barriales, mesas de gestión territorial y efectores sanitarios. En dichos dispositivos participaban vecinos/as, representantes de la UNLP, representantes de proyectos de extensión y de Centros Comunitarios de Extensión Universitaria de la UNLP, de organizaciones sociales, de escuelas, clubes de barrio, responsables de comedores y ollas populares, de efectores sanitarios del primer y del segundo nivel de atención de la zona, de los poderes ejecutivo y legislativo locales, del voluntariado universitario, entre otras. 
Así como el virus, las redes de articulación también se dispersaron radicularmente en los territorios. El supuesto del que partimos es que este acumulado organizativo y multisectorial, que tiene un correlato histórico que ampliaremos más adelante, permite pensar en la experiencia de la pandemia como un proceso de fortalecimiento de la conformación de Redes en Salud territoriales. La sistematización y el estudio de estas experiencias permitirá robustecer una perspectiva de abordaje amplia de la problemática, constituyendo una fuente importante para la conformación de protocolos de acción y el fortalecimiento de las redes. Asimismo, la investigación de estos procesos permitirá ubicarlos en el marco de los grandes debates sanitarios que se desprenden de la pandemia, que circulan en torno a la conformación de un Sistema Integrado de Salud. La pandemia puso en agenda la problemática de la accesibilidad a la salud en nuestro país, por eso creemos que el momento actual es propicio para dar estos debates que implican un cambio profundo en el sistema.

\section{¿Qué entendemos por salud?}

Este artículo presenta una sistematización de los conocimientos acumulados e intenta dar cuenta de los primeros resultados de un proyecto de investigación que se propone analizar las estrategias sanitarias de promoción, prevención y asistencia en barrios populares de La Plata en el marco de la pandemia de COVID-19, desarrolladas a partir de la construcción de redes intersectoriales en salud y dispositivos de organización comunitaria impulsados por los hospitales San Roque y Gutiérrez, la Universidad Nacional de La Plata (UNLP) y las organizaciones comunitarias en barrios de Tolosa y Ringuelet (La Plata), durante el período 2021-2022.

A pesar de los estudios incipientes sobre procesos de organización popular durante la emergencia sanitaria, existe un área de vacancia en investigaciones que indaguen en torno a las redes intersectoriales construidas a partir de la articulación de instituciones gubernamentales, universidad y organizaciones comunitarias en barrios populares en el marco de la pandemia. Por ese motivo nos proponemos, desde una perspectiva transdisciplinaria, analizar esta problemática con aportes de los diversos campos de estudio que conforman el grupo de investigación. 
El marco teórico y epistemológico desde el que nos posicionamos parte de la epidemiología crítica y el abordaje desde una perspectiva de salud colectiva. La epidemiología crítica propone trabajar

por la construcción popular-académica de una sociedad centrada en la vida, la equidad y la plena bioseguridad mediante una investigación intercultural, interdisciplinaria y basada en la comunidad, que busque alimentar un proceso de reforma crítica, cuyo eje estratégico sea el apoyo desde la ciencia epidemiológica a la defensa de los derechos. (Breilh, 2013. p. 23).

Desde esa perspectiva, ponemos en cuestión las definiciones tradicionales de salud, entendiendo que a lo largo de nuestra historia dichas definiciones han tomado distintos sentidos según el paradigma imperante en cada momento histórico. Siguiendo a Casallas Murillo (2017), sostenemos que la salud es un objeto construido y transformado histórica, social y culturalmente y compartimos que «para occidente la comprensión de la salud se ha desarrollado teniendo como referente la enfermedad, es decir, como una situación antagónica a la enfermedad. Esta exclusión explícita del concepto de salud es propia del discurso científico» (p. 400). En ese sentido, nos acercamos al concepto de salud colectiva, en tanto pensamiento crítico y transformador, que

contribuye con el estudio del fenómeno salud/enfermedad en poblaciones en su carácter de proceso social; investiga la producción y distribución de las enfermedades en la sociedad como procesos de producción y reproducción social; procura comprender, finalmente, las formas con que la sociedad identifica sus necesidades y problemas de salud, busca su explicación y se organiza para enfrentarlos (Cueva Dávila, 1989 en Casallas Murillo, 2017, p. 407).

Complementariamente, concebimos a la salud como campo polisémico, que se relaciona directamente con la praxis. Partimos de la mirada del sanitarista Floreal Ferrara, para quien «la salud es la lucha por resolver un conflicto antagónico que quiere evitar que alcancemos el óptimo vital para vivir en la construcción de nuestra felicidad» (Blanco, 2010. p. 2). Esta perspectiva se aleja de considerar a la salud como un estado y enfatiza la idea de lucha: concibe a la salud desde su dinamismo y constante movimiento. Asimismo, resulta 
evidente que Ferrara introduce un anclaje político a la salud, complejizando la mirada sobre los procesos salud-enfermedad-atención-cuidado y ubicando al Estado como el responsable de garantizar su derecho.

Por otra parte, Casallas Murillo (op. cit.) plantea que la determinación social de la salud es una categoría que

observa al objeto de la salud desde un proceso histórico, dialéctico, complejo y multidimensional que abarca la producción de condiciones de salud y de vida, cuyo desarrollo se genera en medio del gran movimiento entre la sociedad y la naturaleza, sometido a su vez a las relaciones productivas y sociales de un sistema de acumulación de poder y cultura (p. 404-405).

La determinación social de la salud reconoce la relación existente entre la política y la ciencia, de modo tal que plantea que la transformación social es el propósito de las investigaciones, perspectiva compartida en este proyecto. Para ello, se requiere la participación de los sujetos para identificar procesos de transformación y producir procesos de emancipación. En ese sentido, en el presente artículo revisaremos modos de organización históricos y locales para luego sistematizar aquellos producidos en pandemia, como parte fundamental del concepto de salud colectiva y determinación social.

Creemos relevante pensar en los aportes de un abordaje integral e intersectorial de los temas y problemas de salud. Integral, en tanto permite analizar a la salud como un proceso en el que inciden diversas variables de diferente tipo (social, cultural, ambiental, habitacional, interpersonal, etc); e intersectorial, en tanto se concibe como una construcción que parte de la participación de diferentes actores -y especialmente de las comunidades-. La intersectorialidad es definida como «la intervención coordinada de instituciones representativas de más de un sector social, en acciones destinadas total o parcialmente a abordar los problemas vinculados con la salud, bienestar y calidad de vida» (Castell-Florit Serrate y Gispert Abreu, 2009, p. 4). Refiere a un acuerdo de trabajo conjunto entre diferentes sectores, en el que cada cual mantiene su especificidad y no necesariamente requiere de la existencia de un enfoque común y transversal a las áreas. La noción de intersectorialidad remite a dos premisas: una plantea la integración entre sectores como vía para la búsqueda de soluciones integrales; la segunda se 
refiere a que la intersectorialidad permite utilizar productivamente los recursos, los saberes y aun los sesgos sectoriales. Cunill $(2005$, p. 1) sostiene que la primera premisa "le asigna un fundamento expresamente político a la intersectorialidad". La articulación intersectorial generada en el marco del trabajo comunitario en Tolosa y Ringuelet no escapa a este componente político y la relación entre los actores vinculados resulta fundamental para pensar integralmente a la salud abordada desde el territorio.

Para poder indagar en esta articulación, retomamos el concepto de redes en salud. Éstas «conectan o vinculan personas», por lo tanto se trata «fundamentalmente de un concepto vincular» y conllevan distintos niveles de profundidad (Rovere, 2016, p. 52). Rovere (op. cit.) plantea que el primer nivel de construcción de una red sería el reconocimiento de un/a otro/a, cuyo valor es la aceptación. El paso siguiente es el conocimiento de quién es ese/a otro/a y qué hace, por lo que su valor es el interés. Luego sigue la colaboración, donde se aportan ayudas esporádicas bajo la lógica de reciprocidad para poder llegar a un nivel más alto de construcción, que es el de cooperación, donde se comparten actividades o recursos bajo el valor de la solidaridad. En un último escalón estaría la asociación, donde se comparten objetivos y proyectos con un alto nivel de confianza.

Por último, para darle un anclaje territorial al concepto de redes en salud, lo conectamos con la perspectiva de la Atención Primaria de la Salud: una estrategia de abordaje «que concibe integralmente los problemas de salud-enfermedad-atención de las personas y del conjunto social, a través de la integración de la asistencia, la prevención de enfermedades, la promoción de la salud y la rehabilitación» (Stolkiner et. al., 2011). Esta conexión nos permite pensar cómo el acumulado histórico de organización comunitaria del territorio en estudio aporta a pensar un abordaje integral de la salud de esa comunidad $y$, asimismo, de qué forma se vio fortalecida esta posibilidad a partir de las acciones generadas durante la pandemia de COVID19.

Antecedentes de organización comunitaria en los barrios de Tolosa y Ringuelet 
Por los objetivos del proyecto, nos interesa señalar especialmente las formas de organización generadas a través del tiempo. Así, en este apartado describiremos de manera sucinta los modos de organización referenciados por vecinos y vecinas, dando cuenta de la presencia de actores diversos. Como sostuvimos en el apartado anterior, creemos que la participación es fundamental para construir salud colectiva desde una mirada integral, es decir, que abarca cuestiones que van más allá del binomio salud-enfermedad y que tienen en cuenta aspectos propios de la determinación social (acceso al hábitat, a la educación, identidades, diversidades culturales y sexo-genéricas, etc).

De acuerdo a relatos de los propios habitantes de los barrios en estudio, las primeras acciones de organización identificadas se vinculan a la agrupación Montoneros, que durante la última dictadura cívico-militar impulsó kermeses, entrega de ropa y comida, festejos de cumpleaños de niñeces y un comedor. En la década de 1980, los vecinos y vecinas destacan la presencia de la organización de la iglesia católica Cáritas y ya en la década de 1990 se mencionan eventos y festividades organizadas por comunidades paraguayas: torneos de fútbol y de truco, asados, bailes, etc. Entrado el siglo XXI, otras organizaciones e instituciones referenciadas son comedores escolares y barriales, centros cristianos metodistas, asociaciones de la sociedad civil y cooperativas de trabajo (Bernat, 2018).

Nos interesa particularmente mencionar el caso de Barrio Nuevo ya que fue construido luego de la inundación del 2 de abril de 2013 e involucra a familias que habitaban antiguos asentamientos de la zona. Alli existió un proceso de organización previo a las mudanzas por la reubicación, en la que articularon vecinos/as, integrantes de organizaciones, referentes de clubes, de movimientos sociales y partidos políticos, entre otros. Una vez mudados/as, las participaciones se vinculaban al pedido de derechos para el barrio (por ejemplo, mudanzas a viviendas definitivas, reclamos por falta de luz y agua, etc). Asimismo, observamos que, al menos hasta mediados de 2017, predominaba una mirada que desvalorizaba los procesos organizativos y los vinculaba negativamente con la participación de partidos políticos, desde una mirada que resaltaba las trayectorias ligadas al esfuerzo individual y familiar. Estimamos que esas percepciones se fueron transformando con el paso del tiempo, a raíz de los procesos suscitados en el país y en la región, que tuvieron incidencia local, y por acontecimientos 
ocurridos en los barrios de la zona, que dieron lugar a la conformación de instancias organizativas con objetivos puntuales, como se verá a continuación.

A partir de 2018 comenzó a forjarse una incipiente organización vecinal con la intención de hacer frente a las principales problemáticas identificadas en el barrio: la ausencia de unión entre sus habitantes y la falta de espacios de contención de los y las jóvenes que derivó, según los análisis de los propios vecinos y vecinas de Barrio Nuevo, en situaciones de consumo problemático de sustancias. Asimismo, a raíz de enfrentamientos armados entre barrios lindantes, se generaron acciones tendientes a la integración de esos espacios, como la organización de torneos de fútbol, de ferias de comidas típicas latinoamericanas, de festejos del día de las niñeces, entre otras (Bernat, 2019). Por último, cabe destacar que durante 2018 y 2019 en conjunto con la Residencia de Medicina General del Hospital Gutiérrez, la Dirección de Redes Intersectoriales en Salud de la UNLP, la Secretaría de Redes en Salud de la Facultad de Ciencias Médicas y organizaciones sociales, como el Refugio del Puente, se llevó a cabo un análisis de situación de salud del barrio y, a partir de sus resultados, se elaboró un proyecto para crear un club y una planta de reciclado, que fue presentado en diciembre de 2019 ante el municipio.

Como dijimos, entendemos que todas estas acciones buscaban resolver problemáticas que forman parte del derecho a la salud desde una mirada integral y que prioriza la determinación social.

\section{Las redes construidas en pandemia}

A partir de lo expuesto anteriormente, podemos afirmar que el inicio de la pandemia encontró al territorio en estudio con un tejido social articulado, que se había organizado ante diversas problemáticas, pero que no incluía a la totalidad de la población que habitaba estos barrios. Consideramos que ese acumulado de participación facilitó la organización de acciones para afrontar la pandemia.

En el marco de la emergencia sanitaria, se propuso un abordaje en conjunto entre la mesa barrial Tolosa-Ringuelet, el voluntariado universitario y los SAPS del Hospital Gutiérrez y 
San Roque. Muchos de los actores que previamente generaban acciones en el barrio se incorporaron a dicha articulación durante la pandemia, lo que aportó a centralizar y unificar las iniciativas y los esfuerzos.

Nos parece importante generar una breve caracterización de los actores involucrados.

Los Servicios de Área Programática y Redes en Salud son servicios hospitalarios creados en octubre de 2020 por la Dirección de Políticas de Atención de la Salud, dependiente de la Dirección Provincial de Hospitales. Su misión principal es promover la articulación de los efectores provinciales con los actores de su área programática para garantizar la accesibilidad de la población al sistema de salud. Los equipos están conformados por profesionales de diferentes disciplinas (medicina, psicología, obstetricia, trabajo social, sociología, comunicación, entre otras) y buscan abordar integralmente las problemáticas de salud.

Con respecto al voluntariado universitario, cabe señalar que en junio del 2020 la UNLP firmó un convenio específico con el Ministerio de Salud de la Provincia de Buenos Aires por el cual más de 2000 voluntarios y voluntarias se sumaron a las acciones dispuestas por las autoridades sanitarias bonaerenses. De ese modo, estudiantes, graduados, docentes y nodocentes de diferentes Facultades (Ciencias Médicas, Odontología, Ciencias Exactas, Periodismo y Comunicación Social, Psicología, Trabajo Social, Humanidades y Ciencias de la Educación, Ingeniería y otras) comenzaron a participar en diferentes líneas del voluntariado, como las que se describirán más adelante. La Dirección de Redes Intersectoriales en Salud de la UNLP participa en su coordinación.

Por su parte -y como ya explicitamos-, la mesa barrial de Tolosa-Ringuelet es un espacio que, desde hace más de diez años, reúne a vecinos y vecinas, representantes de instituciones educativas, comedores, organizaciones sociales y la UNLP para el abordaje y acompañamiento de diversas problemáticas.

En 2020, se realizaron 20 operativos sanitarios en la zona: 8 en Ringuelet y 12 en Tolosa. Entre ellos, destacamos los siguientes barrios: La Unión, Barrio Nuevo, El Mercadito, La Favela, La Bajada, La Isla, El Churrasco y otros puntos del área programática. Estos operativos podrían clasificarse de la siguiente manera: 
- Operativos "Detectar": su principal objetivo fue la identificación de personas que podían tener COVID-19. Se desarrollaron a partir de agendas construidas con la Región Sanitaria número XI, los/as representantes de UNLP en las delegaciones y se incorporan eventualmente postas de vacunación, el Programa Remediar y otras iniciativas complementarias. Las tareas consistían en un relevamiento "puerta a puerta" en el que se preguntaba por síntomas compatibles con COVID-19 y se garantizaba el hisopado en caso de ser necesario.

- Relevamientos sociosanitarios: estas iniciativas contaron con un mayor grado de planificación por parte del efector de salud responsable -en este caso, los hospitales Gutiérrez y San Roque-, mayor nivel de participación de la comunidad y más cantidad de variables a registrar en el "casa por casa". Como mencionamos en la introducción, se registraba a personas embarazadas sin controles de salud, personas con problemas para acceder a métodos anticonceptivos, personas que padecían enfermedades no transmisibles y que también se encontraban sin controles de salud, posibles situaciones de violencia de género, etc.

- Postas de salud: se desarrollaban abordajes más integrales de las comunidades que ya tenían dificultades en su acceso a la salud y que vieron esa situación agravada por el contexto pandémico. En estas postas se realizaron controles de salud de niños/as y embarazadas, se controlaron libretas sanitarias y se llenaron planillas de ANSES, se realizaron talleres sobre distintos temas de prevención de la salud. En el área programática en cuestión, estas postas se llevaron a cabo principalmente en el Club Dardo Rocha.

Asimismo, se conformaron 21 Núcleos Operativos de Emergencia impulsados por la Dirección de Redes Intersectoriales en Salud de la UNLP en los que, como comentamos, se ponían en común información confiable y medidas de cuidado en relación a diferentes problemáticas.

Cabe destacar que en octubre de 2020 comenzó a dictarse en el Club Dardo Rocha -y de la mano del SAPS del Hospital Gutiérrez- la Diplomatura Universitaria en Promoción y Gestión de la Salud Comunitaria, coordinada por la Dirección de Redes Intersectoriales en 
Salud y el Consejo Social de la UNLP. Muchas/os de las y los estudiantes -hoy graduadas/ostambién participaron en los operativos de salud señalados.

En 2021 las acciones implementadas se concentraron en el plan público, gratuito y optativo "Vacunate" en los que se realizaba la inscripción a la campaña de vacunación contra el COVID-19. En esta zona, desde los SAPS del Hospital Gutiérrez y San Roque, en conjunto con el voluntariado y la mesa barrial, se llevaron a cabo jornadas en las que se inscribía a los vecinos y vecinas, se mapeaba esa información y luego se avisaba una vez que recibían el turno para acceder a la vacuna.

Con el inicio de las postas de vacunación itinerantes, estas jornadas incluyen hasta el día de hoy no solo la inscripción a personas pertenecientes a distintos grupos etarios, sino que también se aplican vacunas mediante recorridas casa por casa.

A su vez, se han realizado relevamientos sociosanitarios y postas de salud integrales coordinadas con los SAPS señalados.

En 2021 se realizaron casi 30 operativos abarcando los siguientes barrios: El Mercadito, La Favela, La Bajada, La Unión, El Churrasco, La Isla, Barrio Nuevo y otros puntos del área programática. Además, funciona una posta de salud en el Club Dardo Rocha en la que se realizan controles de niños/as y de adultos/as. 


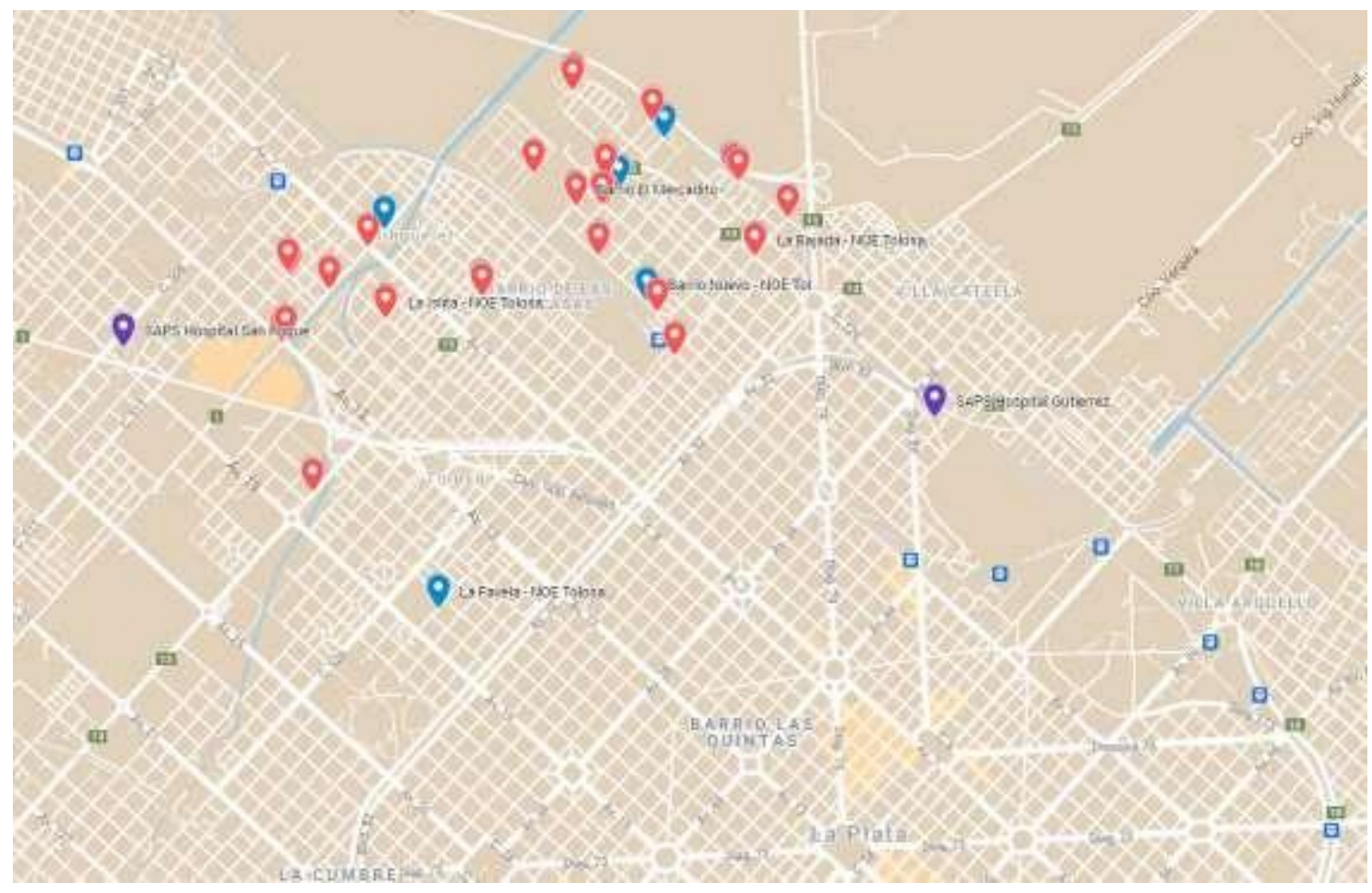

Figura 1: geolocalización de NOEs, operativos y SAPS en los barrios de Tolosa y Ringuelet. Fuente: elaboración propia con aportes de Fonseca, Bernat, Malleville, Sala (2020). Disponible en https://bit.ly/MapaSaludlnvestiga

\section{¿Cómo fue el futuro que vendrá? Aportes desde los territorios para transformar el sistema de salud}

Luego de casi dos años de pandemia, animarnos a imaginar el futuro que vendrá implica un ejercicio teórico y práctico atravesado por incertidumbres y por la certeza de que imperan las contingencias. No obstante, el trabajo realizado durante la emergencia sanitaria nos deja (y nos confirma) algunas pistas sobre ese futuro. 
En primer lugar, reafirmamos la perspectiva de que la salud es un derecho. $\mathrm{Y}$, como todo derecho, se construye, se conquista y/o se lucha para garantizarlo. No hay derecho a la salud sin comunidad organizada y sin un Estado conduciendo ese proceso.

En segundo lugar, con un piso de necesidades y proyectos compartidos, salud es lo que cada comunidad identifica de esa manera. Tal como sugiere el concepto de salud colectiva, tenemos que alejarnos de los intentos por definir las implicancias de esta categoría sin conocer cómo viven, piensan y sienten sus protagonistas.

En tercer lugar, hemos sistematizado diversos aprendizajes preliminares que nos permiten trascender la emergencia sanitaria y nos proponen un modelo -o, al menos, un conjunto de nociones y prácticas- para el abordaje de otras problemáticas de salud. Desde hace tiempo sabemos que uno de los modos más democráticos de abordar estos temas es a partir de la construcción redes intersectoriales, que involucren a actores y actoras diversas, y que se formulen a partir de relaciones que tiendan a la horizontalidad y la equidad. Los procesos suscitados en Ringuelet y Tolosa dan cuenta de ello. Las redes construidas en estos barrios muestran la potencialidad para generar acciones concretas de abordaje de la salud de la población desde una perspectiva amplia, inclusiva y con la comunidad como protagonista.

Durante estos dos años, hemos aprendido que los efectores de salud dan mejores respuestas (más sencillas y efectivas) cuando trabajan de manera articulada. La pandemia dejó en evidencia que la falta de comunicación entre las instituciones del primer nivel y las del segundo nivel de atención vulnera el acceso al sistema de salud de las comunidades y complejiza recorridos ( $y$ accesos) que podrían simplificarse con una mejor articulación. En ese sentido, nos preguntamos: ¿Qué lugar ocupan los centros de atención primaria de la salud en La Plata en 2020 y 2021? ¿Cuál es el alcance del trabajo realizado por los hospitales provinciales en los barrios populares? Sin los accesos posibilitados a partir de las redes intersectoriales construidas, ¿cómo hubiera sido el abordaje de la pandemia? ¿Cuáles son las dimensiones que hay que fortalecer en esta articulación entre niveles para garantizar el acceso al sistema de salud por parte de los sectores más vulnerados?

Las comunidades $-\mathrm{y}$, especialmente, las más vulneradas- se apropian de los efectores de salud cuando se sienten parte y cuando éstos son, en sus prácticas, "de puertas abiertas". 
Además de los NOE, las postas y operativos de salud, ¿qué otros dispositivos podemos proponer para que este aspecto se fortalezca? ¿Cómo es la formación de sus trabajadores/as? ¿Cómo se construyen las relaciones de poder al interior de cada efector? ¿Qué perspectivas, valoraciones y preconceptos se ponen en juego?¿Cómo integramos a la comunidad y sus organizaciones en el debate por la integración del sistema de salud?

La universidad pública desempeña un rol importante en la conformación de una sistema de salud integrado, favoreciendo diálogos y la identificación de necesidades y deseos y poniendo en común los saberes construidos y en construcción para fortalecer el derecho a la salud. También cabe preguntarnos por su papel en la formación de los trabajadores y trabajadoras de la salud: ¿Cuáles son las miradas en torno a la salud que imperan? ¿Cuáles son concretamente los territorios de formación? ¿Qué debates y reflexiones hace falta impulsar desde las unidades académicas para cimentar una salud colectiva e inclusiva?

Los primeros efectos de la pandemia y las transformaciones que nos propone como sociedad comienzan a verse a medida que decantan las experiencias vividas. Las problemáticas de salud encontraron un espacio en la agenda de debate público que hacía mucho tiempo no tenían. Creemos que esto contribuyó a ampliar el marco de discusión, visibilizando una concepción amplia de la salud, no centrada en las enfermedades y concebida como un proceso social integral. En ese sentido, el debate por la construcción de un sistema integrado de salud tomó fuerza y marcó la agenda en este campo de estudio para los próximos años.

Desde nuestra perspectiva, las comunidades tomaron protagonismo en las medidas de promoción y prevención, afrontando con esfuerzos mancomunados y organizados los diversos problemas que la pandemia nos fue planteando como sociedad. La organización popular y la participación comunitaria marcaron el camino para atravesar los peores momentos de ese proceso. Allí encontramos las principales claves para pensar un nuevo modelo de ciudadanía que se identifique con la acción común y el protagonismo popular como pilares para afrontar las problemáticas de salud en los territorios. Es por eso que creemos que el fortalecimiento de las organizaciones sociales y de los vínculos entre el Estado, la sociedad y las universidades resulta un eje fundamental desde donde partir para construir un sistema integrado de salud. 
(1) Este artículo se enmarca en una Beca Salud Investiga (2021-2022) en la categoría de Estudios Múltiples Investigadores del Ministerio de Salud de la Nación. El proyecto se titula: "Estrategias sanitarias de promoción, prevención y asistencia en barrios populares de La Plata en el marco de la pandemia del Covid-19. Análisis de las prácticas impulsadas por los Hospitales San Roque y Gutiérrez, la UNLP y las organizaciones comunitarias". El grupo de investigación que da lugar al proyecto integra profesionales de diferentes campos de saber: psicología, medicina, sociología y comunicación.

(2) Por área programática nos referimos a la delimitación geográfica donde a los efectores de salud les corresponde planificar y ejecutar sus programas sanitarios.

(3) Según el Ministerio de Salud de la Nación, "dada la situación generada por el nuevo coronavirus es necesario establecer estrategias especiales para el abordaje territorial en los Barrios Populares o en áreas definidas donde se detecta o estima un incremento en el número de casos". En este dispositivo, ante un posible caso de COVID-19, se realiza una búsqueda casa por casa de los contactos estrechos de casos confirmados y de todas las personas con síntomas compatibles con COVID-19; se acompaña a las personas al lugar de testeo en el barrio para tomar la muestra; entre otras.

\section{Referencias bibliográficas}

Blanco, B. (19 de abril de 2010). Entrevista inédita a Floreal Ferrara, médico sanitarista recientemente fallecido:"La salud es la solución del conflicto". Página 12.

Bernat, M.S. (2019). "Nacer en determinado lugar te condiciona'. Salud, derecho a la ciudad, crisis y participación en un barrio reubicado". En Revista Ciência \& Saúde Coletiva ${ }^{\circ} 12$ Abrasco, Brasil.

Bernat, M.S. (2018). Transformaciones socio-urbanas y vida cotidiana: el caso de la relocalización de un asentamiento de Ringuelet (2013-2017). Habitar (después de) la inundación. Tesis doctoral. Facultad de Periodismo y Comunicación Social. Universidad Nacional de La Plata. La Plata, Argentina. 
Breilh J. (2013) La determinación social de la salud como herramienta de transformación hacia una nueva salud pública (salud colectiva). Rev. Fac. Nac. Salud Pública 2013; 31(supl 1): S13S27.-Casallas Murillo, AL. La medicina social-salud colectiva latinoamericanas: una visión integradora frente a la salud pública tradicional. Rev Cienc Salud. 2017;15(3):397-408.

Casallas Murillo, AL. La medicina social-salud colectiva latinoamericanas: una visión integradora frente a la salud pública tradicional. Rev Cienc Salud. 2017;15(3):397-408.

Castell-Florit Serrate, P. y Gispert Abreu, E. (2009). La intersectorialidad y el desarrollo de la Salud Pública en Cuba. Revista Cubana de Salud Pública, 35(1).

Cunill Grau, N. (2005) "La intersectorialidad en el gobierno y gestión de la política social". Ponencia presentada en el X Congreso Internacional del CLAD sobre la reforma del Estado y de la Administración Pública. Santiago de Chile.

CLACSO. (2020). Economías populares en la pandemia. Cartografía provisoria en tiempos de aislamiento y crisis global. Grupo de Trabajo CLACSO- Economía popular: mapeo teórico y práctico.

Fonseca, M, Bernat, S., Malleville, S. y Sala, J. (2020). Informe anual. Dispositivos, impacto, actores, territorios y desafíos. Abordaje integral de la pandemia por COVID-19. Dirección de Redes Intersectoriales en Salud de la Universidad Nacional de La Plata. La Plata: UNLP. Disponible en: https://drive.google.com/file/d/1c2aKm1gJn2RS5qsOJ4sQ7o1yLF4kfPn9/view

Rovere, M. (2016). Redes en salud: los grupos, las instituciones, la comunidad. Córdoba: El Ágora.

Sanchís, N. (2020). El cuidado comunitario en tiempos de pandemia... y más allá. Argentina: Asociación Lola Mora.

Stolkiner, A; Comes, Y.; Garbus, P. (2011). Alcances y potencialidades de la Atención Primaria de la Salud en Argentina. Ciência \& Saúde Coletiva, 16 (6), 2807-2816. 
Question/Cuestión, Vol. 3, № 70

Diciembre 2021

ISSN 1669-6581

Vommaro, P. (2020). Durante y después de la pandemia: dimensiones sociales, políticas y económicas. En: Vommaro, P. (2020). Alerta global. Políticas, movimientos sociales y futuros en disputa en tiempos de pandemia. Argentina: Consejo Latinoamericano de Ciencias Sociales. 